\title{
Linear and Nonlinear Heart Rate Variability Analysis in Gonadal Dysgenesis (Swyer Syndrome): A Case Report
}

Valdelias Xavier Pereira, ${ }^{\circledR}$ Tatiana Dias de Carvalho, ${ }^{2}{ }^{\circledR}$ Marcos Antonio Marinovic Junior, ${ }^{1}$ Alex Rey Norberto, ${ }^{10}$ José Maria Soares Júnior, ${ }^{\circledR}{ }^{\circledR}$ Vitor Engrácia Valenti, ${ }^{3}{ }^{\circledR}$ Isabel Cristina Esposito Sorpreso ${ }^{1}{ }^{\circledR}$

Faculdade de Medicina da Universidade de São Paulo,' São Paulo, SP - Brazil Universidad Nacional de La Matanza, ${ }^{2}$ San Justo, Buenos Aires - Argentina Universidade Estadual Paulista, ${ }^{3}$ São Paulo, SP - Brazil

\begin{abstract}
Swyer syndrome is one of the disorders of sexual differentiation. Previous studies have demonstrated increased sympathetic activity with heart rate variability (HRV) analysis with decreasing estradiol levels. One patient presented a pure 46, XY gonadal dysgenesis with female phenotype. Cardiac autonomic modulation was assessed through HRV analysis while at rest. This research analyzed linear and nonlinear indexes. HRV analysis showed reduced parasympathetic and global modulation with an apparent increase in sympathetic tone and a loss of HR fractal dynamics toward correlated behavior, characterized by low entropy and high determinism of time series.
\end{abstract}

\section{Introduction}

Swyer syndrome is one of the disorders of sexual differentiation (DSD), a pure gonadal dysgenesis with karyotype 46, XY, the presence of two digenetic gonads or gonads streak. and devoid of germinative elements. The clinical presentations are female phenotype with typical female external genitalia, primary amenorrhea and persistent hypergonadotropic hypogonadism, normal stature, and absence of somatic malformations. ${ }^{1}$

Previous studies have demonstrated increased sympathetic activity with heart rate variability (HRV)

\section{Keywords}

Autonomic Nervous System; Estrogen, Replacement Therapy; Amenorrhea; Gonadectomy; Heart Rate; Cardiovascular Diseases/complications. analysis with decreasing estradiol levels. However, the clinical and therapeutic implication of autonomic impairment is still unclear. ${ }^{1}$ This study reports on the autonomic behavior of HRV in Swyer Syndrome, but the report exempts itself from an in-depth review.

\section{Case Report}

A 48-year-old married Brazilian woman participated in this research in the Endocrine Gynecology and Menopause Clinic the São Paulo University Medical School. Patient follow-up began in 1983, at which time the patient received a diagnosis of gonadal dysgenesis in her adolescence. This case report was approved by the ethics committee of the Faculdade de Medicina da Universidade de São Paulo (FMUSP) (Protocol n ${ }^{\circ}$ 2.368.076), and the patient's anonymity and consent were guaranteed.

After the primary amenorrhea diagnosis, followed by the gonadal dysgenesis diagnosis, the patient was treated with estradiol valerate and levonorgestrel ( $2 \mathrm{mg}+0.25 \mathrm{mg}) /$ day. At age 21, she went through a bilateral oophorectomy procedure. She reported no other hospitalizations, preexisting diseases, or use of other regular medication. Her gynecological examination showed female genitalia with trophism and pubic hair development, both ageappropriate, and no injuries.

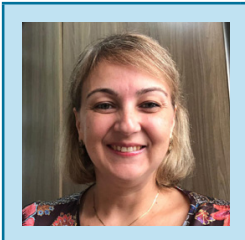

Isabel Cristina Esposito Sorpreso, MD, PhD Associate Professor of the Discipline of Gynecology Department of Obstetrics and Gynecology, Faculty of Medicine, University of São Paulo (FMUSP)

Mailing Address: Tatiana Dias de Carvalho

Florencio Varela, 1903. Postal Code: B1754JEC, San Justo, Buenos Aires - Argentina

E-mail: carvalho.td1@gmail.com 
She reported no chronic or infectious gynecological diseases. She denied alcohol or drug abuse, but had a history a smoking. The general physical examination showed good general condition, ruddy, hydrated, afebrile, respiratory rate of $19 / \mathrm{min}$, normal blood pressure, body mass index of $33.33 \mathrm{~kg} / \mathrm{m}^{2}$. Cardiac, pulmonary, abdominal, and biochemical exams showed no relevant findings.

Cardiac autonomic modulation was assessed through HRV analysis. RR interval recordings were obtained by a validated heart rate receiver, ${ }^{2}$ placed on the chest, with the patient at rest for $20 \mathrm{~min}$. HRV analysis was performed as proposed by Godoy et al., ${ }^{3}$ using linear (time and frequency domains) and nonlinear methods. ${ }^{4}$ Additionally, geometric indexes, Poincaré and recurrence plots, entropy-based techniques, and Detrended Fluctuations Analysis ${ }^{5}$ (DFA) were analyzed (Table 1).

\section{Discussion}

This study found signs of reduced parasympathetic and global modulation with an apparent increase in the sympathetic tone and a loss of HR fractal dynamics toward correlated behavior, characterized by low entropy and high determinism in a time series.

Our study subject had been using hormonal therapy (HT) (natural estrogen and second-generation progestogen) since having been diagnosed with amenorrhea. Previous studies ${ }^{1,6}$ have evaluated the effect of sex hormones and distinct interventions on cardiac autonomic modulation. It is known that menopausal women present a lower HRV when compared with women who are not in menopause, ${ }^{6}$ and that $\mathrm{HT}^{7}$ and exercise $^{8}$ can apparently improve cardiac autonomic modulation. Liu et al., ${ }^{9}$ investigated the role of estrogen in gender-related autonomic differences and found that during the postmenopausal period, the vagal and sympathetic activities were lower and higher, respectively.

Effects of oophorectomy on cardiac autonomic modulation revealed an imbalance in the regulation of the cardiovascular system, with a decrease in cardiac vagal modulation and an increase in sympathetic activity. ${ }^{10}$ Since our patient underwent a bilateral oophorectomy procedure, this case report also incorporates important

Table 1 - HRV linear and nonlinear indices of the Swyer Syndrome patient

\begin{tabular}{|c|c|c|c|}
\hline Linear methods & \multicolumn{3}{|c|}{ Nonlinear methods } \\
\hline Time domain & \multicolumn{3}{|c|}{ Recurrence plot } \\
\hline Mean RRi (ms) & 722.1 & \multirow{10}{*}{$\begin{array}{l}\text { Lmean (beats) } \\
\text { Lmax (beats) } \\
\text { REC (\%) } \\
\text { DET (\%) } \\
\text { ShanEn }\end{array}$} & \multirow{4}{*}{16.37} \\
\hline SDNN (ms) & 21.3 & & \\
\hline Mean HR (1/min) & 83.16 & & \\
\hline RMSSD (ms) & 9.5 & & \\
\hline Geometrics & & & 988 \\
\hline RR tri & 6.711 & & 41.30 \\
\hline TINN (ms) & 105.0 & & 99.43 \\
\hline SD1 (ms) & 6.7 & & 3.668 \\
\hline SD2 (ms) & 29.3 & & \\
\hline SD1/SD2 & 0.228 & & \\
\hline \multicolumn{2}{|l|}{ Frequency domain } & \multicolumn{2}{|c|}{ Other } \\
\hline $\mathrm{LF}\left(\mathrm{ms}^{2}\right)$ & 118 & ApEn & 1.226 \\
\hline $\mathrm{HF}\left(\mathrm{ms}^{2}\right)$ & 19 & SampEn & 1.227 \\
\hline LF (nu) & 86.1 & DFA alpha1 & 1.416 \\
\hline HF (nu) & 13.9 & DFA alpha2 & 1.007 \\
\hline LF/HF & 6.191 & alpha1/alpha2 & 1.406 \\
\hline $\begin{array}{l}\text { RRi: RR interval, SD } \\
\text { frequency, nu: norma } \\
\text { in continuous RRis, } \\
\text { Mean line length; Lm } \\
\text { Sample entropy; DFA }\end{array}$ & $\begin{array}{l}\text { leviation } \\
\text { tri: tria } \\
\text { deviatio } \\
\text { ength; } R \\
\text { ictuatio }\end{array}$ & $\begin{array}{l}\text { square of successi } \\
\text { n, SD1: standard } \\
\text { between short and } \\
\text { n: Shannon Entro }\end{array}$ & $\begin{array}{l}\text { frequency, HF high } \\
\text { teneous variability } \\
\text { intervals. Lmean: } \\
\text { te entropy; SampEn: }\end{array}$ \\
\hline
\end{tabular}


endocrine features, which, along with genetic features, were both important to understand the modification of cardiovascular risk.

In linear methods, we found lower parasympathetic indices (RMSSD and HF) than the mean values of previous studies. ${ }^{1} \mathrm{LF}$ and LF/HF were higher, which could be related to an increase in the sympathetic tone. ${ }^{4,5}$ SDNN, which presents global modulation, was lower than the mean values of the same studies, which could be due to reduced parasympathetic activity.,

As no specific studies about Swyer Syndrome with geometric and nonlinear indexes were found, our results have been compared with those from a menopause study ${ }^{8}$ with a similar HT. All of this study's geometric indexes, both parasympathetic (SD1) and global indexes (RRtri, TINN, SD2 and SD1/SD2), were reduced. Regarding nonlinear indices, the present study shows higher values. In the DFA analysis, values close to 1.5 are associated with signs of strongly correlated behavior, ${ }^{5}$ while our results pointed to a strongly correlated behavior with possible loss of fractal properties. Autonomic conditions characterized by sympathetic predominance or by the reduction in global modulation often present a correlated behavior and decrease in complexity. ${ }^{5}$

Sample Entropy is used to evaluate the complexity of the physiological time-series signals. Approximate Entropy is applied to quantify the amount of regularity fluctuations upon time-series data and is linked with vagal modulation and HF. Higher values indicate more complex data. Shannon entropy quantifies the degree of complexity of the signal's sample distribution. In physiological conditions, where there is a loss of time series complexity, the recurrence of points in the system increases. Their diagonals, such as Lmean, express the similarity of system behavior in two distinct time sequences. ${ }^{5}$ In our patient, in general, nonlinear indices showed a loss of HR fractal dynamic toward correlated behavior, characterized by low entropy and high determinism in the time series. REC and DET values were very similar to linear pattern values, typical of reduced complexity. ${ }^{11}$

Our results were then compared with those from Rismini et al., ${ }^{12}$ who evaluated the sympathovagal balance in transsexuals. Their data demonstrated that male-to-female transsexuals (a supposedly similar genetic condition, $X Y$, with HT) displayed significantly lower sympathetic and parasympathetic activities than did the controls, which could be mediated by the effect of hexogen estrogens, since these subjects do not have other protective factors.
To the best of our knowledge, this is the first study in the literature describing HRV nonlinear indices in Swyer Syndrome. However, this study has some limitations. First, because this is a case report, there are limitations regarding comparisons with previous literature. Secondly, it is possible that the individual's BMI and smoking history could alter the HRV parameters. Nevertheless, our study findings point out possible negative effects of hypoestrogenism, gonadectomy, and aging in subjects with DSD, as well as the importance of HT to improve autonomic regulation during one's lifetime.

\section{Conclusion}

HRV analysis in Swyer syndrome showed reduced parasympathetic and global modulation with an apparent increase in sympathetic tone and a loss of HR fractal dynamic toward correlated behavior, characterized by low entropy and high determinism in a time series. Our findings suggest that individuals with Swyer syndrome may have increased cardiovascular risk despite HT.

\section{Author Contributions}

Conception and design of the research: VX Pereira, ICE Sorpreso. Acquisition of data: VX Pereira, AR Norbeto. Analysis and interpretation of the data: TD Carvalho, VE Valenti. Statistical analysis: TD Carvalho, VE Valenti. Writing of the manuscript: MA Marinovic Junior e JM Soares Júnior. Critical revision of the manuscript for intellectual content: ICE Sorpreso.

\section{Acknowledgments}

We are grateful to the patient, who gave her informed consent for publication.

\section{Potential Conflict of Interest}

No potential conflict of interest relevant to this article was reported.

\section{Sources of Funding}

There were no external funding sources for this study.

\section{Study Association}

This article is part of the thesis of post doctoral submitted by Valdelias Xavier Pereira, from Faculdade de Medicina da Universidade de São Paulo. 


\section{Ethics approval and consent to participate}

This study was approved by the Ethics Committee of the Faculdade de Medicina da Universidade de São Paulo (FMUSP) under the protocol number 2.368.076. All the

\section{References}

1. Goldmeier S, De Angelis K, Rabello Casali K, Vilodre C, ConsolimColombo F, Belló Klein A et al. Cardiovascular autonomic dysfunction in primary ovarian insufficiency: clinical and experimental evidence. Am J Transl Res. 2013, 6(1):91-101.

2. Gamelin FX, Berthoin S, Bosquet L. Validity of the polar S810 heart rate monitor to measure RR intervals at rest. Med Sci Sports Exerc. 2006; 38(5):887-93.

3. Godoy MF, Takakura IT, Correa PR. Relevância da análise do comportamento dinamico não linear (Teoria do Caos) como elemento prognostico de morbidade e mortalidade em pacientes submetidos a cirurgia de revascularização miocárdica. Arq Cienc Saude. 2005 12(4):167-71.

4. Vanderlei LCM, Pastre CM, Hoshi RA, Carvalho TD, Godoy MF. Noções básicas de variabilidade da frequência cardíaca e sua aplicabilidade clínica. Braz J cardiovasc Surg. 2009; 24: 205-17.

5. Huikuri HV, Makikallio TH, Perkiomaki J. Measurement of heart rate variability by methods based on nonlinear dynamics. J Electrocardiol. 2003; 36:95-9.

6. Jurca R, Church TS, Morss GM, Jordan AN, Earnest CP. Eight weeks of moderate-intensity exercise training increases heart rate variability in sedentary postmenopausal women. Am Heart J. 2004; 147:e21. procedures in this study were in accordance with the 1975 Helsinki Declaration, updated in 2013. Informed consent was obtained from all participants included in the study.

7. Yildirir A, Kabakci G, Yarali H, Aybar F, Akgul E, Bukulmez O, et al. Effects of hormone replacement therapy on heart rate variability in postmenopausal women. Ann NoninvasElectrocardiol. 2001; 6(4):280-4.

8. Rezende Barbosa MPDC, Vanderlei LCM, Neves LM, Takahashi C, Torquato PRDS, Fortaleza ACS, et al. Impact of functional training on geometric indices and fractal correlation property of heart rate variability in postmenopausal women. Ann Noninvas Electrocardiol. 2018; 23(1): 1-9.

9. Liu CC, Kuo TB, Yang CC. Effects of estrogen on genderrelated autonomic differences in humans. Am J Physiol Heart Circ Physiol. 2003; 285(5):H2188-93.

10. Mercuro G, Podda A, Pitzalis L, Zoncu S, Mascia M, Melis GB, Rosano GM. Evidence of a role of endogenous estrogen in the modulation of autonomic nervous system. Am J Cardiol. 2000; 85(6):787-9.

11. Takakura IT, Hoshi RA, Santos MA, Pivatelli FC, Nóbrega JH, Guedes DL, et al. Recurrence Plots: a New Tool for Quantification of Cardiac Autonomic Nervous System Recovery after Transplant. Braz J Cardiovasc Surg 2017;32(4):245-52.

12. Resmini E, Casu M, Patrone V, Rebora A, Murialdo G, Minuto F, et al. Sympathovagal imbalance in transsexual subjects. J Endocrinol Invest. 2008: 31(11):1014-9. 